OPEN ACCESS

Edited by:

Holger Andreas Russ,

University of Colorado Anschutz

Medical Campus, United States

Reviewed by:

Rosa Gasa,

Institut de Recerca Biomèdica August

Pi i Sunyer (IDIBAPS), Spain

Senta Georgia,

Children's Hospital of Los Angeles,

United States

*Correspondence:

Shimon Efrat

sefrat@tauex.tau.ac.ll

Specialty section:

This article was submitted to Diabetes: Molecular Mechanisms,

a section of the journal

Frontiers in Endocrinology

Received: 05 October 2020 Accepted: 02 December 2020

Published: 19 January 2021

Citation:

Efrat S (2021) Epigenetic Memory:

Lessons From iPS Cells

Derived From Human $\beta$ Cells.

Front. Endocrinol. 11:614234. doi: 10.3389/fendo.2020.614234

\section{Epigenetic Memory: Lessons From iPS Cells Derived From Human $\beta$ Cells}

\author{
Shimon Efrat* \\ Department of Human Molecular Genetics and Biochemistry, Sackler School of Medicine, Tel Aviv University, Tel Aviv, Israel
}

Incomplete reprogramming of somatic cells into induced pluripotent stem cells (iPSCs) may be responsible for the heterogeneity in differentiation capacity observed among iPSC lines. It remains unclear whether it results from stochastic reprogramming events, or reflects consistent genetic or cell-of-origin differences. Some evidence suggests that epigenetic memory predisposes iPSCs to enhanced differentiation into the parental cell type. We investigated iPSCs reprogrammed from human pancreatic islet $\beta$ cells (BiPSCs), as a step in development of a robust differentiation protocol for generation of $\beta$-like cells. BiPSCs derived from multiple human donors manifested enhanced and reproducible spontaneous and induced differentiation towards insulin-producing cells, compared with iPSCs derived from isogenic non- $\beta$-cell types and fibroblast-derived iPSCs (FiPSCs). Genome-wide analyses of open chromatin in BiPSCs and FiPSCs identified thousands of differential open chromatin sites (DOCs) between the two iPSC types. DOCs more open in BiPSCs (Bi-DOCs) were significantly enriched for known regulators of endodermal development, including bivalent and weak enhancers, and FOXA2 binding sites. BiDOCs were associated with genes related to pancreas development and $\beta$-cell function. These studies provide evidence for reproducible epigenetic memory in BiPSCs. Bi-DOCs may provide clues to genes and pathways involved in the differentiation process, which could be manipulated to increase the efficiency and reproducibility of differentiation of pluripotent stem cells from non- $\beta$-cell sources.

\section{Keywords: Epigenetic memory, Islet $\beta$ Cells, pluripotent stem cell differentiation, ATAC-seq, Foxa2}

\section{INCOMPLETE REPROGRAMMING INTO IPSCS}

Reprogramming of somatic cells into induced pluripotent stem cells (iPSCs) using Yamanaka's combination of four transcription factors (OCT4, SOX2, KLF4, and c-MYC, together termed OSKM) (1) has opened new avenues for in-vitro generation of multiple human differentiated cell types for disease modeling, drug screening, and regenerative medicine. Initially iPSCs derived from multiple cell types have been expected to manifest similar differentiation capacities, resembling those of human embryonic stem cells (ESCs). However, accumulated experience has shown a great heterogeneity in differentiation capacity among iPSC lines. This heterogeneity is thought to result from incomplete reprogramming. 
The mechanisms activated by ectopic expression of OSKM in somatic cells, which result in reprogramming to pluripotency, are only partly understood. This prolonged process, lasting several weeks, involves multiple events, including silencing of somatic cell genes and activation of pluripotency-associated genes. The low efficiency of OSKM-mediated reprogramming is thought to reflect the stochastic nature of these complex events. Only a small fraction of cells acquire pluripotency, as judged by accepted assays (e.g. differentiation into cells from the three embryonic germ layers in embryoid bodies (EB), and teratoma formation assay). OSKM factors function by binding to chromatin regions and inducing their remodeling, thereby activating or repressing gene expression. Evidence suggests that broad epigenetic changes are among early key events of the reprogramming process (2).

The epigenetic landscape, shaped by DNA methylation and histone modifications, is critical for maintaining cell identity. Erasing cell-specific patterns of epigenetic modifications, and replacing them with pluripotency patterns, are central to cell reprogramming to pluripotency. Ample evidence supports the incomplete and varying erasure of the original epigenetic marks in both mouse and human iPSC lines. However, it remains unclear whether these variations represent consistent patterns, based on the cell type of origin, or genetic differences among donors, or reflect stochastic differences caused by low efficiency of the reprogramming mechanisms (Table 1). While epigenetic memory may not necessarily affect gene expression patterns in iPSCs (3), likely due to missing transcription factors, it is expected to affect differentiation capacity towards specific cell fates. This may limit some applications of iPSCs, but at the same time may predispose iPSCs to enhanced differentiation into the parental cell type, thereby facilitating generation of cells for specific uses.

\section{IPSC DIFFERENCES RELATED TO THE CELL TYPE OF ORIGIN}

\section{Mouse iPSCS}

Early comparison of mouse iPSCs derived from bone marrow progenitor cells, dermal fibroblasts, and neural progenitor cells, identified residual DNA methylation signatures characteristic of the somatic tissue of origin (4). These differences favored iPSC differentiation towards the donor cell type, while restricting

TABLE 1 | Possible sources of iPSC variability.

\begin{tabular}{ll}
\hline $\begin{array}{l}\text { Possible } \\
\text { source of iPSC } \\
\text { variability }\end{array}$ & \multicolumn{1}{c}{ Expected phenotype } \\
\hline $\begin{array}{l}\text { Stochastic } \\
\text { reprogramming } \\
\text { events }\end{array}$ & $\begin{array}{l}\text { Differences among clones from a single cell type of origin from } \\
\text { a single donor }\end{array}$ \\
$\begin{array}{l}\text { Donor-related } \\
\text { Cell type of } \\
\text { origin }\end{array}$ & $\begin{array}{l}\text { Similarity among clones from multiple cell types of origin from } \\
\text { a single donor; differences among iPSCs from multiple donors } \\
\text { Similarity among iPSCs from the same cell type of origin from } \\
\text { multiple donors }\end{array}$
\end{tabular}

alternative cell fates. These results were contrasted to the methylation and differentiation patterns of nuclear-transferderived pluripotent stem cells, which were more similar to those of ESCs. Similar findings were reported in early-passage iPSCs obtained from mouse fibroblasts, hematopoietic and myogenic cells, which exhibited distinct epigenetic patterns (5). These patterns were reflected in different transcriptional profiles of the iPSCs, and in their differentiation efficiency into embryoid bodies (EBs) and hematopoietic cell types. These differences were eroded with iPSC passaging, suggesting that epigenetic memory was a transient phenomenon. iPSCs derived from mouse neonatal cardiomyocytes (CMs) were also shown to differentiate toward CMs more efficiently than fibroblast-derived iPSCs (FiPSCs) or mouse ESCs (6).

\section{Human iPSCs}

A number of reports documented similar epigenetic memory in human iPSCs. Kim et al. compared DNA methylation profiles and differentiation potential of iPSCs derived from human umbilical cord blood and neonatal keratinocytes (7). They identified distinct genome-wide DNA methylation patterns in iPSCs derived from each cell type, resulting from both incomplete erasure of tissue-specific methylation and aberrant de-novo methylation. These differences did not disappear upon extended passaging. Ohi et al. observed that human iPSCs generated from hepatocytes, skin fibroblasts, and melanocytes, retained some transcriptional characteristics of the original cells at low passages, which could be partially explained by incomplete promoter DNA methylation (8). They noticed that incompletely silenced genes tended to be isolated from other genes that were repressed during reprogramming, indicating that silencing of isolated genes may be less efficient. Global DNA methylation analyses of iPSCs reprogrammed from human cornea limbal epithelial stem cells (LESC) showed gene methylation patterns similar to those of the parental cells, and different from those of FiPSCs (9). Upon differentiation, LESC-derived iPSCs expressed higher levels of LESC markers, compared with FiPSCs.

\section{IPSC DIFFERENCES RELATED TO DONORS AND STOCHASTIC VARIABILITY}

In contrast to these findings, which associated epigenetic and differentiation differences among iPSCs with their cell type of origin, other studies supported a donor-related or stochastic basis for these differences. In one such study (10), whole-genome profiling of DNA methylation in five human iPSC lines derived from adipocytes and fibroblasts (including 3 subclones of a single line) identified over 1,000 differentially methylated sites, most of them associated with CG islands and genes, indicating stochastic interclone reprogramming variability. Two other studies $(11,12)$ surveyed 16-18 iPSC lines each, derived from fibroblasts and peripheral blood cells of four human donors in each study. Both studies concluded that the majority of transcriptional and DNA methylome differences among iPSCs, as well as differences in their differentiation capacity towards the hematopoietic cell 
lineage, could be attributed to the donor, rather than the cell type of origin, indicating that genetic differences among donors can result in reproducible reprogramming differences. Finally, two studies, which analyzed differences in gene expression patterns in human iPSCs, concluded that genetic differences between individual donors were the major cause of transcriptional variation between lines. One of these studies (13) compared 25 iPSC lines from four donors and three tissues. The second study compared two human ESC lines with genetically matched iPSCs derived from fibroblasts differentiated from each ESC line (14). Their findings showed reproducible gene expression patterns among each ESC line and three iPSC clones derived from it, and variations compared with the other ESC line and its iPSC derivatives. The study concluded that ESCs and iPSCs exhibited similar gene expression patterns, and that the donor genetic background was responsible for transcriptional variations among pluripotent stem cell lines.

The donor genetic background may also influence the differentiation capacity of ESC lines. However, in contrast to the relatively large numbers of iPSC lines that have been generated by multiple laboratories, data on differentiation of ESC lines accumulated to date is based on a small number of established ESC lines commonly used by all research groups. This limited number, which is a result of ethical barriers to generation of new ESC lines, does not allow proper comparisons of variations among ESC lines in differentiation potential into specific cell lineages.

\section{EPIGENETIC MEMORY IN IPSCS DERIVED FROM HUMAN $\beta$ CELLS}

Directed in-vitro differentiation of iPSCs into $\beta$-like cells is a promising approach for generation of abundant insulinproducing cells for cell therapy of diabetes, disease modeling and drug screening. Despite significant progress (15-21), current differentiation protocols result in cells with heterogeneous and immature phenotype, and suffer from low efficiency and variability among iPSC lines. In a step towards developing a more robust differentiation protocol, we investigated iPSCs reprogrammed from human pancreatic islet $\beta$ cells (BiPSCs). This approach depended on lineage tracing of human $\beta$-cellderived (BCD) cells within the heterogeneous cell population in cultures of isolated human islets (22). The stable genetic label allowed positive identification of the $\beta$-cell origin of individual iPSC clones, that otherwise would be difficult to distinguish from iPSCs derived from non- $\beta$-cell types present in the expanded islet cell culture. Initial studies of four BiPSC lines derived from $\beta$ cells of three nondiabetic human donors established their pluripotency, as judged by standard assays (23). Nevertheless, chromatin immunoprecipitation (ChIP) analyses showed that the levels of histone $\mathrm{H} 3$ acetylation, a hallmark of open chromatin structure, at the INS and PDX1 loci in BiPSCs were similar to those found in human islets and BCD cells, and significantly higher than those in FiPSCs, as well as in two iPSC lines derived from isogenic islet non- $\beta$ cells (termed
PiPSCs) from two of the donors. BiPSCs also exhibited significantly lower DNA methylation levels, characteristic of transcribed genes, in genes expressed in human islet cells, such as $P D X 1$, compares with FiPSCs. Despite the open chromatin marks, $\beta$-cell genes were not expressed in BCD and BiPS cells.

The epigenetic memory of BiPSCs was associated with higher expression levels of PDX1, FOXA2, and INS transcripts following spontaneous differentiation into EBs and teratomas, compared with those derived from FiPSCs and PiPSCs, and an enhanced induced differentiation capacity into insulin-producing cells in mice transplanted with BiPSC-derived endocrine progenitors, following the protocol of Kroon et al. (24). The epigenetic phenotype and differentiation capacity of BiPSCs were reproducible among the four lines obtained from three human donors, and appeared stable within the passage range analyzed (passages 10-20).

To identify genes and pathways, which may be responsible for the enhanced and reproducible differentiation capacity of BiPSCs, we performed a global analysis of chromatin sites differentially open in BiPSCs, compared with FiPSCs, using an Assay for Transposase Accessible Chromatin with highthroughput sequencing (ATAC-seq) (25). For this analysis we generated five new BiPSC lines from three nondiabetic donors, which were compared to five FiPSC lines from two nondiabetic donors. All these lines passed pluripotency assays, and EBs generated from BiPSCs at passages 9-12 showed enhanced spontaneous expression of FOXA2, PDX1, and INS, compared with those derived from FiPSCs (25), similarly to the four BiPSC lines in the initial study.

Despite high overall similarity in open chromatin between the two iPSC types, the ATAC-seq analysis identified thousands of significantly differential open chromatin (DOC) sites between BiPSCs and FiPSCs, most of which were more open in BiPSCs (Bi-DOCs). Bi-DOCS overlapped gene regulatory elements known to be involved in development, such as weak enhancers (marked by H3K4me1) and bivalent enhancers and promoters (marked by $\mathrm{H} 3 \mathrm{~K} 27 \mathrm{me} 3$ ), especially near genes involved in endodermal development, such as FOXA2 and its target genes, and pancreas development, such as $P D X 1$, and $N K X 2-2$, as well as genes expressed in mature $\beta$ cells, such as INS. The ATAC-seq data for these four genes (FOXA2, PDX1, NKX2-2, and INS) was validated by $\mathrm{H} 3 \mathrm{~K} 4 \mathrm{me} 3 \mathrm{ChIP}$ analysis, which found higher levels of this open chromatin mark in the promoter regions of all four genes in BiPSCs, compares with FiPSCs. These findings could explain the enhanced expression of these genes in EBs generated from BiPSCs, compared with those derived from FiPSCs.

The relevance of Bi-DOCs to differentiation towards islet cells was analyzed by comparing directed differentiation of BiPSCs and FiPSCs into definitive endoderm (DE) and pancreatic progenitor (PP) cells according to the protocol of Rezania et al. (16). Global transcriptome analyses by RNA-seq identified 567 protein-coding genes expressed at higher levels in BiPSC-derived DE, compared with FiPSC-derived DE, and 181 genes expressed at higher levels in BiPSC-derived PP, compared with FiPSCderived PP (25). These genes were significantly enriched for genes mapping near Bi-DOCs. Among genes expressed at higher 
levels in BiPSC at both stages, the most prominent was estrogen receptor 1 (ESR1; 3.5-fold and 53.4-fold higher in DE and PP, respectively) and several of its target genes. 17 $\beta$-estradiol (E2) has been shown to protect mouse $\beta$ cells from apoptosis by signaling through estrogen receptor (ER) $\alpha$ encoded by ESR 1 (26). ER $\alpha$ activity has been reported to increase Neurog3 expression and $\beta$-cell proliferation in a mouse model of pancreas partial duct ligation, and during mouse islet development (27). It has been suggested to regulate endocrine lineage specification through downregulation of $\mathrm{NOTCH}$ signaling. Thus, inclusion of E2 in the culture medium at key stages of the in-vitro differentiation protocol may increase its efficiency and reproducibility.

\section{CONCLUSION}

Overall, the analyses of Bi-DOCs support the existence of reproducible epigenetic memory in BiPSCs. The association between Bi-DOCs and gene expression levels at early stages of the in-vitro differentiation protocol provides a plausible explanation for the enhanced differentiation capacity of BiPSCs into the $\beta$-cell lineage, compared with pluripotent stem cells from a non- $\beta$-cell source. Both chromatin structure and differentiation capacity were reproducible in a combined

\section{REFERENCES}

1. Takahashi K, Yamanaka S. Induction of pluripotent stem cells from mouse embryonic and adult fibroblast cultures by defined factors. Cell (2006) 126:663-76. doi: 10.1016/j.cell.2006.07.024

2. Watanabe A, Yamada Y, Yamanaka S. Epigenetic regulation in pluripotent stem cells: a key to breaking the epigenetic barrier. Philos Trans $R$ Soc Lond B Biol Sci (2013) 368:20120292. doi: 10.1098/rstb.2012.0292

3. Cahan P, Daley GQ. Origins and implications of pluripotent stem cell variability and heterogeneity. Nat Rev Mol Cell Biol (2013) 14:357-68. doi: 10.1038/nrm3584

4. Kim K, Doi A, Wen B, Ng K, Zhao R, Cahan P, et al. Epigenetic memory in induced pluripotent stem cells. Nature (2010) 467:285-90. doi: 10.1038/ nature09342

5. Polo JM, Liu S, Figueroa ME, Kulalert W, Eminli S, Tan KY, et al. Cell type of origin influences the molecular and functional properties of mouse induced pluripotent stem cells. Nat Biotechnol (2010) 28:848-55. doi: 10.1038/nbt.1667

6. Rizzi R, Di Pasquale E, Portararo P, Papait R, Cattaneo P, Latronico MV, et al. Post-natal cardiomyocytes can generate iPS cells with an enhanced capacity toward cardiomyogenic re-differentation. Cell Death Differ (2012) 19:116274. doi: $10.1038 /$ cdd.2011.205

7. Kim K, Zhao R, Doi A, Ng K, Unternaehrer J, Cahan P, et al. Donor cell type can influence the epigenome and differentiation potential of human induced pluripotent stem cells. Nat Biotechnol (2011) 29:1117-9. doi: 10.1038/nbt.2052

8. Ohi Y, Qin H, Hong C, Blouin L, Polo JM, Guo T, et al. Incomplete DNA methylation underlies a transcriptional memory of somatic cells in human iPS cells. Nat Cell Biol (2011) 13:541-9. doi: 10.1038/ncb2239

9. Sareen D, Saghizadeh M, Ornelas L, Winkler MA, Narwani K, Sahabian A, et al. Differentiation of human limbal-derived induced pluripotent stem cells into limbal-like epithelium. Stem Cells Transl Med (2014) 3:1002-12. doi: 10.5966/sctm.2014-0076

10. Lister R, Pelizzola M, Kida YS, Hawkins RD, Nery JR, Hon G, et al. Hotspots of aberrant epigenomic reprogramming in human induced pluripotent stem cells. Nature (2011) 471:68-73. doi: 10.1038/nature09798

11. Kyttälä A, Moraghebi R, Valensisi C, Kettunen J, Andrus C, Pasumarthy KK, et al. Genetic Variability Overrides the Impact of Parental Cell Type and number of nine BiPSC lines from six human islet donors in two separate studies. Bi-DOCs may provide clues to genes and pathways involved in the differentiation process, which could be manipulated to increase the efficiency and reproducibility of differentiation of pluripotent stem cells from other sources. Such manipulations could include activation of candidate genes using CRISPR-on approaches or small-molecule compounds.

\section{AUTHOR CONTRIBUTIONS}

The author confirms being the sole contributor of this work and has approved it for publication.

\section{ACKNOWLEDGMENTS}

This research in my laboratory was supported by the Juvenile Diabetes Research Foundation (JDRF), the Israel Science Foundation, and the Innovative Medicines Initiative of the European Union Seventh Framework Program. Human islets for these studies were provided through the JDRF ECIT Islets for Basic Research Program and the Integrated Islet Distribution Program.

Determines iPSC Differentiation Potential. Stem Cell Reports (2016) 6:200-12. doi: 10.1016/j.stemcr.2015.12.009

12. Burrows CK, Banovich NE, Pavlovic BJ, Patterson K, Gallego Romero I, Pritchard JK, et al. Genetic Variation, Not Cell Type of Origin, Underlies the Majority of Identifiable Regulatory Differences in iPSCs. PloS Genet (2016) 12: e1005793. doi: 10.1371/journal.pgen.1005793

13. Rouhani F, Kumasaka N, de Brito MC, Bradley A, Vallier L, Gaffney D. Genetic background drives transcriptional variation in human induced pluripotent stem cells. PloS Genet (2014) 10:e1004432. doi: 10.1371/ journal.pgen.1004432

14. Choi J, Lee S, Mallard W, Clement K, Tagliazucchi GM, Lim H, et al. A comparison of genetically matched cell lines reveals the equivalence of human iPSCs and ESCs. Nat Biotechnol (2015) 33:1173-81. doi: 10.1038/nbt.3388

15. Pagliuca FW, Millman JR, Gürtler M, Segel M, Van Dervort A, Ryu JH, et al. Generation of functional human pancreatic $\beta$ cells in vitro. Cell (2014) 159:428-39. doi: 10.1016/j.cell.2014.09.040

16. Rezania A, Bruin JE, Arora P, Rubin A, Batushansky I, Asadi A, et al. Reversal of diabetes with insulin-producing cells derived in vitro from human pluripotent stem cells. Nat Biotechnol (2014) 32:1121-33. doi: 10.1038/nbt.3033

17. Russ HA, Parent AV, Ringler JJ, Hennings TG, Nair GG, Shveygert M, et al. Controlled induction of human pancreatic progenitors produces functional beta-like cells in vitro. EMBO J (2015) 34:1759-72. doi: 10.15252/ embj.201591058

18. Nair GG, Liu JS, Russ HA, Tran S, Saxton MS, Chen R, et al. Recapitulating endocrine cell clustering in culture promotes maturation of human stem-cellderived $\beta$ cells. Nat Cell Biol (2019) 21:263-74. doi: 10.1038/s41556-018-0271-4

19. Veres A, Faust AL, Bushnell HL, Engquist EN, Kenty JH, Harb G, et al. Charting cellular identity during human in vitro $\beta$-cell differentiation. Nature (2019) 569:368-73. doi: 10.1038/s41586-019-1168-5

20. Velazco-Cruz L, Song J, Maxwell KG, Goedegebuure MM, Augsornworawat P, Hogrebe NJ, et al. Acquisition of Dynamic Function in Human Stem CellDerived $\beta$ Cells. Stem Cell Reports (2019) 12:351-65. doi: 10.1016/ j.stemcr.2018.12.012

21. Hogrebe NJ, Augsornworawat P, Maxwell KG, Velazco-Cruz L, Millman JR. Targeting the cytoskeleton to direct pancreatic differentiation of human 
pluripotent stem cells. Nat Biotechnol (2020) 38:460-70. doi: 10.1038/s41587020-0430-6

22. Russ HA, Bar Y, Ravassard P, Efrat S. In vitro proliferation of cells derived from adult human beta cells revealed by cell-lineage tracing. Diabetes (2008) 57:1575-83. doi: 10.2337/db07-1283

23. Bar-Nur O, Russ HA, Efrat S, Benvenisty N. Epigenetic memory and preferential lineage-specific differentiation in induced pluripotent stem cells derived from human pancreatic islet beta cells. Cell Stem Cell (2011) 9:17-23. doi: 10.1016/j.stem.2011.06.007

24. Kroon E, Martinson LA, Kadoya K, Bang AG, Kelly OG, Eliazer S, et al. Pancreatic endoderm derived from human embryonic stem cells generates glucose-responsive insulin-secreting cells in vivo. Nat Biotechnol (2008) 26:443-52. doi: 10.1038/nbt1393

25. Thurner M, Shenhav L, Wesolowska-Andersen A, Bennett AJ, Barrett A, Gloyn AL, et al. Genes associated with pancreas development and function maintain open chromatin structure in iPSCs generated from human pancreatic beta cells. Stem Cell Rep (2017) 9:1395-405. doi: 10.1016/ j.stemcr.2017.09.020
26. Le May C, Chu K, Hu M, Ortega CS, Simpson ER, Korach KS, et al. Estrogens protect pancreatic beta-cells from apoptosis and prevent insulin-deficient diabetes mellitus in mice. Proc Natl Acad Sci USA (2006) 103:9232-37. doi: 10.1073/pnas.0602956103

27. Yuchi Y, Cai Y, Legein B, De Groef S, Leuckx G, Coppens V, et al. Estrogen receptor $\alpha$ regulates $\beta$-cell formation during pancreas development and following injury. Diabetes (2015) 64:3218-28. doi: 10.2337/db14-1798

Conflict of Interest: The author declares that the research was conducted in the absence of any commercial or financial relationships that could be construed as a potential conflict of interest.

Copyright $\odot 2021$ Efrat. This is an open-access article distributed under the terms of the Creative Commons Attribution License (CC BY). The use, distribution or reproduction in other forums is permitted, provided the original author(s) and the copyright owner(s) are credited and that the original publication in this journal is cited, in accordance with accepted academic practice. No use, distribution or reproduction is permitted which does not comply with these terms. 